Argonne

ANL/NE-14/7

\title{
User Manual for EXODUS II Mesh Converter
}

Nuclear Engineering Division 


\begin{abstract}
About Argonne National Laboratory
Argonne is a U.S. Department of Energy laboratory managed by UChicago Argonne, LLC under contract DE-AC02-06CH11357. The Laboratory's main facility is outside Chicago, at 9700 South Cass Avenue, Argonne, Illinois 60439. For information about Argonne and its pioneering science and technology programs, see www.anl.gov.
\end{abstract}

\title{
DOCUMENT AVAILABILITY
}

Online Access: U.S. Department of Energy (DOE) reports produced after 1991 and a growing number of pre-1991 documents are available free via DOE's SciTech Connect (http://www.osti.gov/scitech/)

\author{
Reports not in digital format may be purchased by the public from the \\ National Technical Information Service (NTIS): \\ U.S. Department of Commerce \\ National Technical Information Service \\ 5301 Shawnee Rd \\ Alexandra, VA 22312 \\ www.ntis.gov \\ Phone: (800) 553-NTIS (6847) or (703) 605-6000 \\ Fax: (703) 605-6900 \\ Email: orders@ntis.gov \\ Reports not in digital format are available to DOE and DOE contractors from the \\ Office of Scientific and Technical Information (OSTI): \\ U.S. Department of Energy \\ Office of Scientific and Technical Information \\ P.O. Box 62 \\ Oak Ridge, TN 37831-0062 \\ www.osti.gov \\ Phone: (865) 576-8401 \\ Fax: (865) 576-5728
}

\begin{abstract}
Disclaimer
This report was prepared as an account of work sponsored by an agency of the United States Government. Neither the United States Government nor any agency thereof, nor UChicago Argonne, LLC, nor any of their employees or officers, makes any warranty, express or implied, or assumes any legal liability or responsibility for the accuracy, completeness, or usefulness of any information, apparatus, product, or process disclosed, or represents that its use would not infringe privately owned rights. Reference herein to any specific commercial product, process, or service by trade name, trademark, manufacturer, or otherwise, does not necessarily constitute or imply its endorsement, recommendation, or favoring by the United States Government or any agency thereof. The views and opinions of document authors expressed herein do not necessarily state or reflect those of the United States Government or any agency thereof, Argonne National Laboratory, or UChicago Argonne, LLC.
\end{abstract}




\section{User Manual for EXODUS II Mesh Converter}

prepared by

E. R. Shemon and C. W. Attaway

Nuclear Engineering Division, Argonne National Laboratory

August 15, 2014 


\begin{abstract}
A mesh conversion program has been developed to convert EXODUS II-formatted finite element meshes to the ascii format used by the PROTEUS-SN neutronics code developed at Argonne National Laboratory. This manual contains instructions, usage examples, and methodology for the mesh converter.

The PROTEUS-SN neutronics code requires a finite element mesh input file in one of several accepted formats (ascii, nemesh, pntmesh, or bgpmesh). None of these file formats are directly produced by conventional finite element file modeling codes, therefore format conversion is required. Currently, most finite element meshes are prepared using the Cubit program developed by Sandia National Laboratory. Cubit can export finite element meshes in a variety of formats including the portable EXODUS II format. EXODUS II files are binary files with NetCDF data storage structures designed to handle large object-oriented arrays. While a specialized EXODUS II application programming interface (API) exists to query EXODUS II data, the general NetCDF API can also be used for the same purpose.

The described program improves upon a previous tool which had limited flexibility and performance. Notably, the new converter successfully converts EXODUS II files generated with more recent versions of Cubit (version 13+) and has no limitation on mesh size (total number of vertices or elements). The converter queries data from the EXODUS II file using NetCDF API commands, reads the data in the file, reorganizes it, and writes it to the ascii file format used by PROTEUS-SN.
\end{abstract}




\section{TABLE OF CONTENTS}

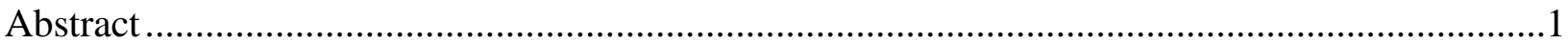

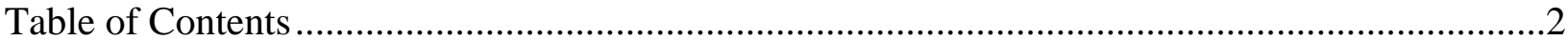

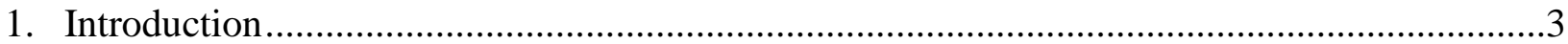

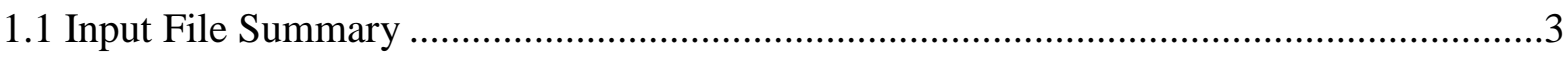

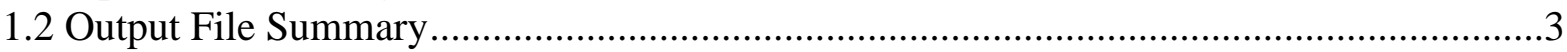

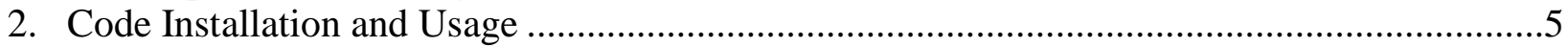

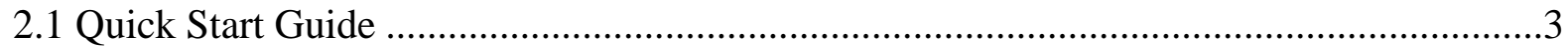

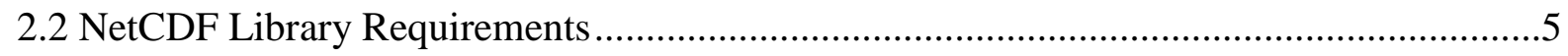

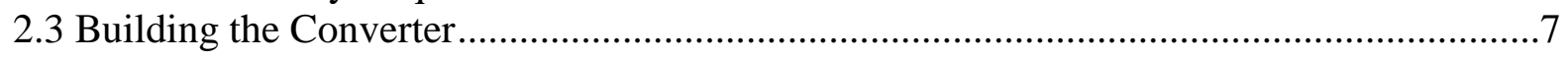

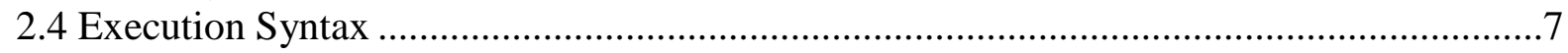

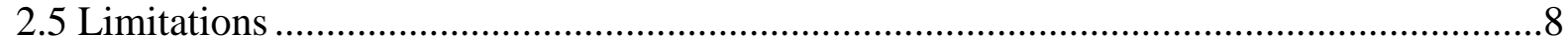

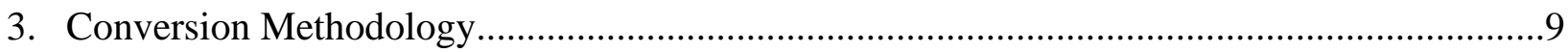

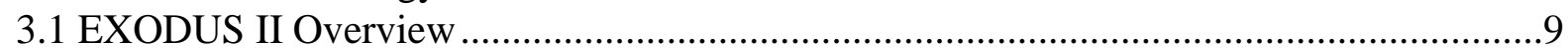

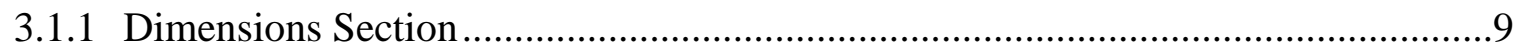

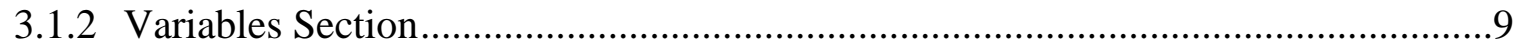

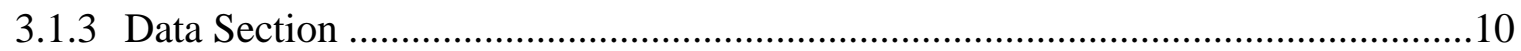

3.2 Conversion Process .............................................................................................. 11

3.2.1 Command Line Input and Output File Creation.............................................11

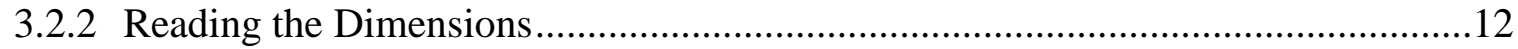

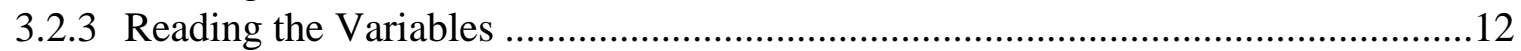

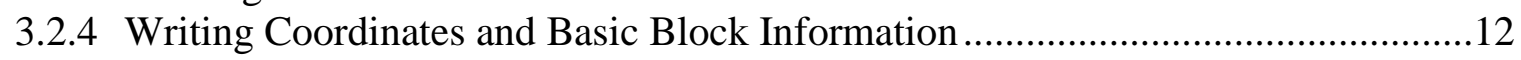

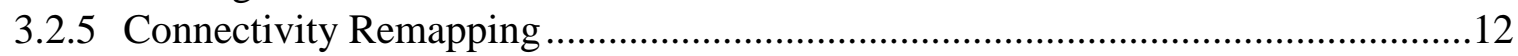

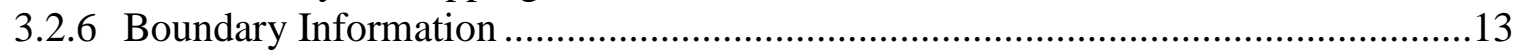

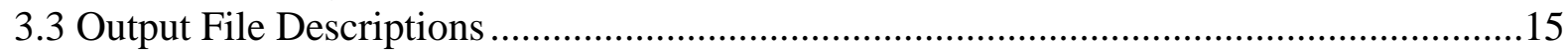

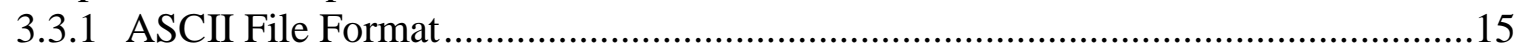

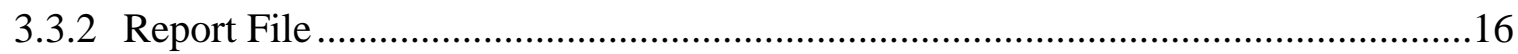

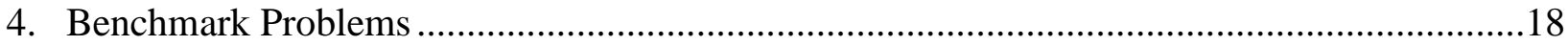

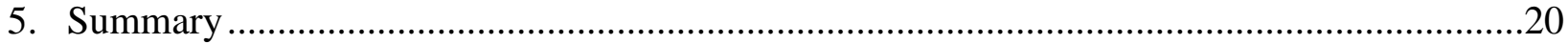

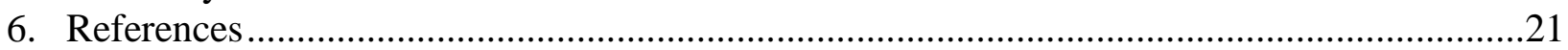

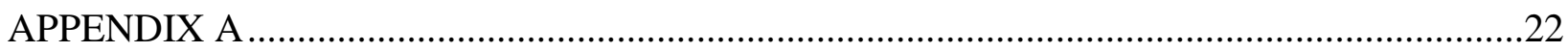

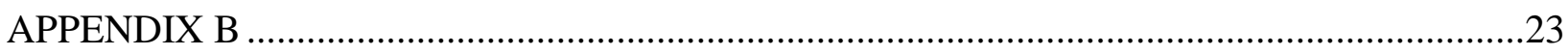




\section{Introduction}

This chapter describes the basic functionality of the EXODUS II mesh converter program, whose purpose is to convert EXODUS II finite element meshes to the ascii format used by the PROTEUS transport code developed at Argonne National Laboratory. The program is written in $\mathrm{C}++$ and depends on a C++-enabled NetCDF [3] library to access and interpret data collected from the input EXODUS II mesh.

\subsection{Input File Summary}

The program requires input in the form of one or more EXODUS II finite element meshes with proper extension type (".e" or ".exo"). The EXODUS II files must contain data following the EXODUS II naming conventions and must not be empty.

The Cubit meshing program [4] developed by Sandia National Laboratory is typically used to create meshes in EXODUS II format and has been routinely used to for PROTEUS-SN neutronics analysis. This converter program is compatible with EXODUS II meshes exported from Cubit versions 12+. Note that Cubit 13+ EXODUS II meshes have slightly different keywords for the vertex coordinates, and this difference is handled inside the converter automatically without user intervention.

\subsection{Output File Summary}

For each EXODUS II input mesh, two output files are produced: the ascii-formatted mesh for PROTEUS and an informative report file. Both files are generated in the same directory as the input EXODUS II mesh and follow a natural naming convention (input file Sample.e yields output files Sample.ascii and Sample_REPORT.txt). The report file is provided as a user convenience to ensure accurate conversion and enable debugging - the conversion procedure is echoed in this file during the conversion process, including any errors encountered.

\subsection{Quick Start Guide}

The Makefile included with the distribution must be modified to properly compile the code. First, modify the NETCDF_DIR variable to point to the local installation of NetCDF (version 4.0 or later, with $\mathrm{C}++/ \mathrm{C}$ support). The converter requires the NetCDF API to function - refer to Section 2.1 for NetCDF installation tips. For full verification capability, also modify the CUBIT_12X, CUBIT_13X, and PROTEUS_X variables to point to the Cubit 12 , Cubit 13, and PROTEUS-SN executables on the local machine. If any of these variables are left blank, the corresponding extended verification tests will not be performed, but the converter can still be compiled and tested. Figure 1 lists the variables in the Makefile which should be modified.

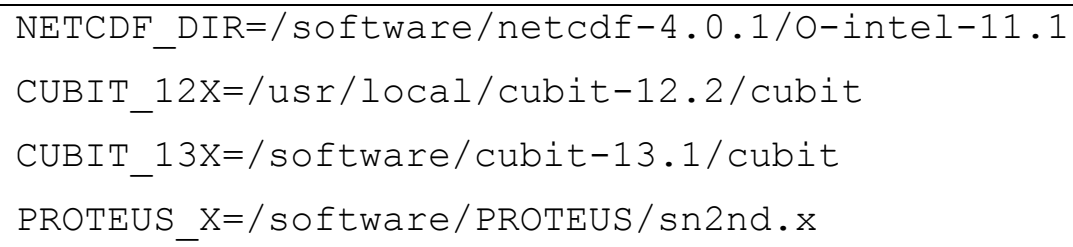


Figure 1. Makefile variables to be modified for installing and testing converter.

Next, the code can be compiled using "make" to create the executable, and "make check" to test basic performance. Optional extended testing with Cubit and PROTEUS are available by typing "make verify" after also providing paths to Cubit and PROTEUS executable locations in CUBIT_12X, CUBIT_13X, and PROTEUS_X variables as shown in the figure above.

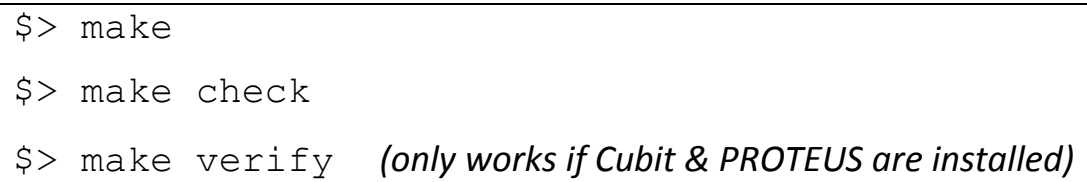

Figure 2. Commands to compile and test the converter.

To run a job, simply type "ExodusConverter.x MyMesh.e" where MyMesh.e is the name (or path) of any EXODUS II file you would like to convert. Upon successful conversion, MyMesh.ascii and MyMesh_REPORT.txt appear in the same directory as MyMesh.e.

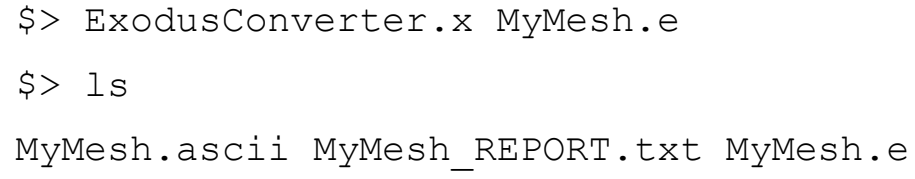

Figure 3. Example command line usage of the converter.

The file MyMesh_REPORT.txt should be examined briefly for any errors. The file MyMesh.ascii can be directly used with PROTEUS if no errors were found. 


\section{Code Installation and Usage}

This section describes how to install and use the EXODUS II finite element mesh converter.

\subsection{NetCDF Library Requirements}

The EXODUS II mesh converter has a dependency on the NetCDF library. It uses the NetCDF API to query data from EXODUS II files which are actually specialized NetCDF data files. As mentioned in the previous section, the NETCDF_DIR variable in the Makefile must point to a local installation of the NetCDF library, version 4.0+, with $C$ and $\mathrm{C}++$ capabilities enabled. The NetCDF library must be built with the same compiler that will be used for the EXODUS II Converter. The user is recommended to confirm the compiler version and $\mathrm{C}++$ support by running the 'nc-config' utility with '-all' option located in the bin directory of the NetCDF installation. This command will list the NetCDF configuration information and capabilities as shown in Figure 4.

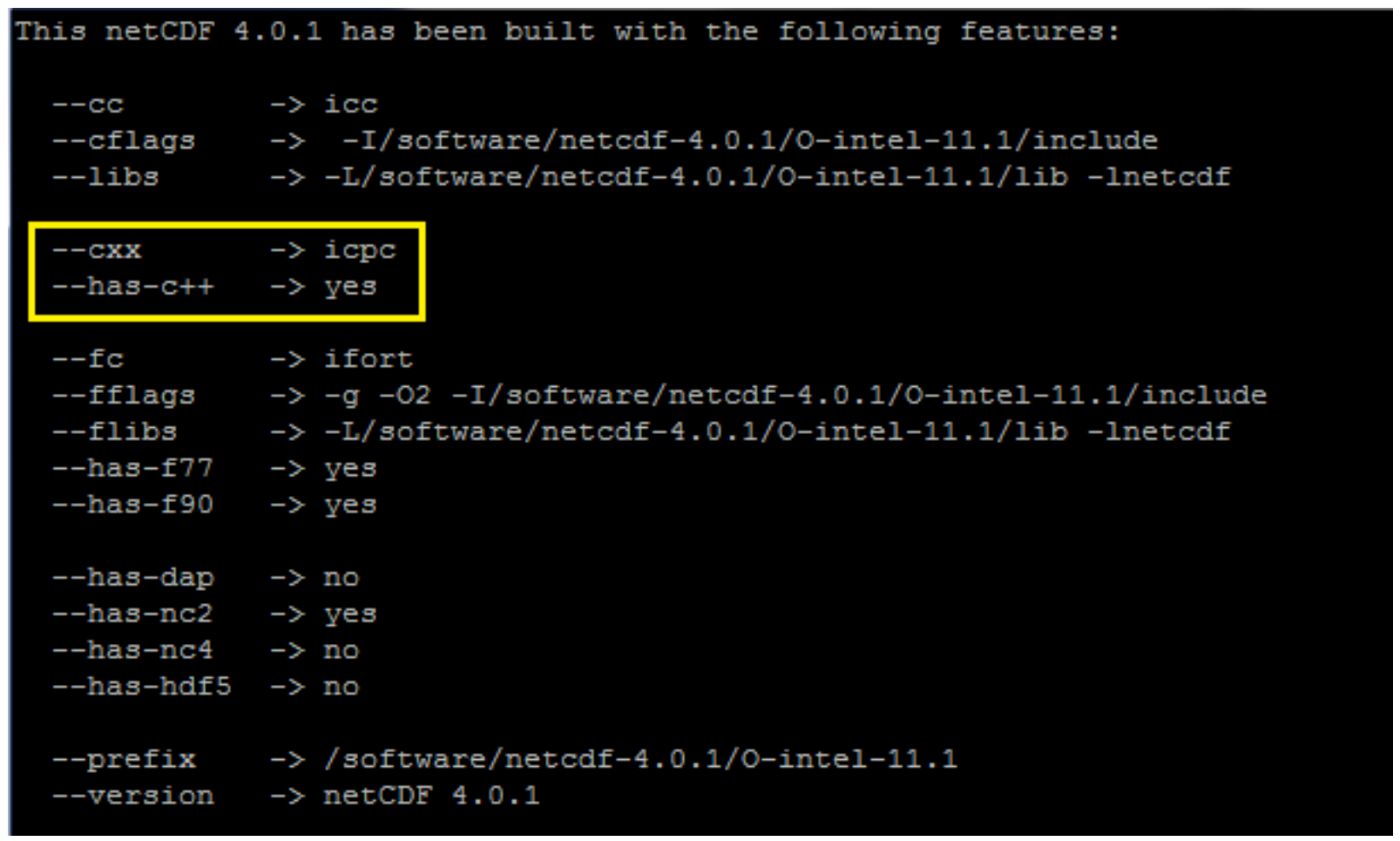

Figure 4. The NetCDF build specifications can be viewed running "nc-config -all" to display the current build information and the library flags.

The NetCDF package can be downloaded from the web (we recommend version 4.0.1, available at www.unidata.ucar.edu/downloads/netcdf/ftp/netcdf-4.0.1.tar.gz). Newer versions (4.2+) are available but require supplemental installations for $\mathrm{C}++$ support. The recommended tarball contains everything needed by the converter in a single package. To configure, we recommend using the 
options in Figure 5, where the compilers should be consistent with those you plan to use for the EXODUS II converter. The "--disable-netcdf-4 --disable-dap-remote-tests" options disable extended NetCDF functionalities that may require additional dependencies that are unnecessary for the converter.

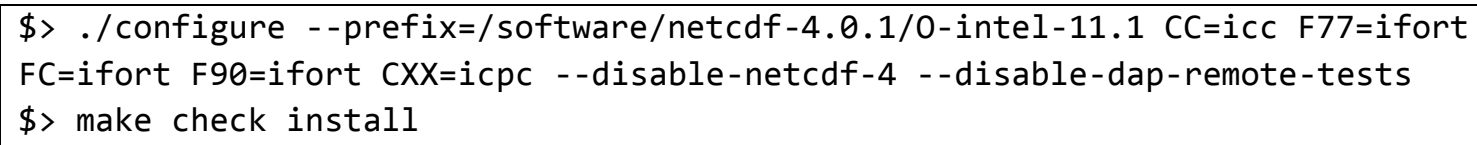

Figure 5. Recommended NetCDF configuration options for NetCDF 4.0 or 4.1 versions.

Table 1 lists the NetCDF function interfaces used by the converter (taken from NetCDF 4.0.1). If using a version of NetCDF past 4.0.1, check whether the function interfaces have changed as the converter will not work properly without updating the function interfaces to match the NetCDF API.

Table 1. NetCDF library functions used in the EXODUS II mesh converter (taken from NetCDF 4.0.1).

\begin{tabular}{|c|c|}
\hline Function Interface & Purpose \\
\hline nc_open(Filename, ReadWriteAccess, \&ncid) & $\begin{array}{l}\text { Opens the EXODUS II file and gives it the } \\
\text { handle ncid }\end{array}$ \\
\hline nc_inq_dimid(ncid, const char name, \&id) & $\begin{array}{l}\text { Returns the address (id) of a given } \\
\text { dimension variable (name) }\end{array}$ \\
\hline nc_inq_dimname (ncid, id, \&characterarray) & $\begin{array}{l}\text { Returns the name (characterarray) of a } \\
\text { dimension variable, given its address (id) }\end{array}$ \\
\hline nc_inq_dimlen(ncid, id, \&length) & $\begin{array}{l}\text { Returns the value of the dimension variable } \\
\text { (length) given its address (id) }\end{array}$ \\
\hline nc_inq_varid(ncid, const char name, \&id) & $\begin{array}{l}\text { Returns the address (id) of a variable, given } \\
\text { its name (name) }\end{array}$ \\
\hline nc_inq_varname (ncid, id, \&characterarray) & $\begin{array}{l}\text { Returns the name of a variable } \\
\text { (characterarray), given its address (id) }\end{array}$ \\
\hline $\begin{array}{l}\text { nc_get_att_text(ncid, id, const char } \\
\text { name, char * text[\#] ) }\end{array}$ & $\begin{array}{l}\text { Returns the value (text) of an attribute } \\
\text { (name) associated with a variable, given its } \\
\text { address (id) }\end{array}$ \\
\hline nc_get_var_int (ncid, id, int * data [\#]) & $\begin{array}{l}\text { Retrieve entire data array (data) from a } \\
\text { variable data array, given its address (id) }\end{array}$ \\
\hline
\end{tabular}


nc_get_var1_int(ncid, id, $\{0,0\}$, sdatapt) Retrieve a single data point (data) from a variable data array, given its address $(i d)$

\subsection{Building the Converter}

In order to compile the converter tool, the user must first edit the NETCDF_DIR variable in the Makefile to point to the local NetCDF installation location as in Figure 1. The other variables are optional to compile and perform basic tests with the converter, but required for the most rigorous verification tests.

Once the Makefile has been properly modified to point to the NetCDF installation, executing the command "make" will invoke compilation and produce the executable "ExodusConverter. $x$ " in the current directory.

Next, the command "make check" should be issued to ask the converter to perform a series of benchmark tests. Each of these tests has a unique feature that will test all file variables that may be present in an EXODUS II mesh from CUBIT. After converting each of the provided EXODUS II files, the contents and size of the provided reference outputs and the new outputs are compared. Any differences denote a failed test and will be written to the command line immediately. Once all tests have been run and compared, the bench directories are cleaned of all files created during the testing.

Finally, the command "make verify" should be issued to generate meshes with different versions of Cubit, convert the meshes from EXODUS II to ascii, and test the meshes in PROTEUS-SN. This step can be skipped if no versions of Cubit are installed locally.

The command "make clean" removes the object file, executables, and any old verification test files.

\subsection{Execution Syntax}

After successfully building and testing, the program can be used to convert any EXODUS II mesh to the PROTEUS-SN ascii file format. Simply type the name of the program followed by the paths to as many inputs as needed as in Figure 6.

\$> ExodusConverter.x MyMesh.exo ThisMesh.e /path/to/ThatMesh.e

Figure 6. Example usage of converter with multiple input files.

The input files must have the correct EXODUS II file extension (".e" or ".exo") for proper conversion. Note that if paths to the input files are specified such as /path/to/my/Exodusmesh.e, the resulting ascii file will be produced in the same directory, /path/to/my/Exodusmesh.ascii. 


\subsection{Limitations}

The converter supports Cubit element types which are also supported by PROTEUS. Therefore, some element types available within Cubit are not valid element types for the converter and PROTEUS. When generating a mesh with Cubit, use only the following element types: BAR, BAR2, BAR3, TRI, TRI3, TRI6, QUAD, QUAD4, QUAD8, QUAD9, TETRA, TETRA4, TETRA10, HEX, HEX8, HEX10. In particular, ensure that the EXODUS II mesh does not contain SHELL or TRISHELL element types which are not supported by PROTEUS-SN or the converter. SHELL or TRISHELL element types are typically assigned by default if the user fails to specify an element type - thus the user should carefully check that they have assigned all element blocks properly. 


\section{Conversion Methodology}

This chapter contains a description of the code methodology and is intended for an advanced user who wishes to modify the converter or understand exactly how the conversion is performed. We focus on describing EXODUS II finite element files created by Cubit 12+. EXODUS II files created by other software may have slightly different formats.

\subsection{EXODUS II Overview}

The EXODUS II file type is binary, portable, and cannot be read in a standard text editor. To read the contents of an EXODUS II file, the NetCDF utility "ncdump" can be used to dump the content to plain text. Each EXODUS II mesh file contains three important data sections: "dimensions", "variables", and "data".

\subsubsection{Dimensions Section}

The "dimensions" section of an EXODUS II file defines values for the following "dimension" variables. These dimensions are used to declare arrays appearing later in the file.

Table 2. EXODUS II dimensions given in mesh file.

\begin{tabular}{|l|l|}
\hline EXODUS II Dimension & Definition \\
\hline len_string & Max length of a string \\
\hline num_dim & Number of dimensions in the mesh 1, 2, 3 \\
\hline num_nodes & Total number of nodes (vertices) in the mesh \\
\hline num_elem & Total number of elements in the mesh \\
\hline num_el_blk & Total number of element blocks in the mesh \\
\hline num_side_sets & Total number of sidesets in the mesh \\
\hline num_side_ss1 & Number of elements that have a surface belonging to sideset 1 (etc.) \\
\hline num_el_in_blk1 & Number of elements in block 1 (etc.) \\
\hline num_nod_per_el1 & Number of nodes per element in block 1 (etc.) \\
\hline
\end{tabular}

\subsubsection{Variables Section}

The "variables" section of an EXODUS II file contains the declaration of data arrays including their dimensions and their "attributes". Note the dimensions of each of the variables were defined in the "dimensions" section. The only data contained in this section is the element type of each block, and it is given as an attribute of the connectivity array for each block. 
Table 3. EXODUS II variables declared in file.

\begin{tabular}{|c|c|}
\hline EXODUS II Variable & Definition \\
\hline char coor_names(num_dim, len_string) & character array holding the names of the dimensions \\
\hline $\begin{array}{l}\text { int ss_prop1(num_side_sets) } \\
\text { ss_prop1: name = "ID" }\end{array}$ & $\begin{array}{l}\text { Integer array holding the "prop1" attribute for all } \\
\text { the sidesets. "prop1" in this case is defined as the } \\
\text { "ID", so the array ss_prop1 holds the "ID" for each } \\
\text { sideset. }\end{array}$ \\
\hline int elem_ss1(num_side_ss1) & $\begin{array}{l}\text { Integer array defining the elements which have a } \\
\text { surface in sideset } 1 \text { (etc.) }\end{array}$ \\
\hline int side_ss1(num_side_ss1) & $\begin{array}{l}\text { Integer array defining the reference surface on each } \\
\text { element belong to sideset } 1 \text { (etc.) }\end{array}$ \\
\hline $\begin{array}{l}\text { int eb_prop1(num_el_blk) } \\
\text { eb_prop1: name = "ID" }\end{array}$ & $\begin{array}{l}\text { Integer holding the "prop1" attribute for the } \\
\text { element blocks. "prop1" in this case is defined as } \\
\text { "ID", so the array eb_prop1 holds the "ID" for each } \\
\text { element block. }\end{array}$ \\
\hline $\begin{array}{l}\text { int connect1(num_el_in_blk1, } \\
\text { num_nod_per_el1) } \\
\text { connect1:elem_type = "QUAD8" }\end{array}$ & $\begin{array}{l}\text { Integer array holding the connectivity for element } \\
\text { block } 1 \text { (etc.). The element block has the attribute } \\
\text { "elem_type" which in this example is "QUAD8". }\end{array}$ \\
\hline $\begin{array}{l}\text { [Cubit } 12 \text { and earlier convention] } \\
\text { double coord(num_dim, num_nodes) }\end{array}$ & $\begin{array}{l}\text { Real array holding the coordinates of the vertices, in } \\
\text { order of all } X \text {, all } Y \text {, and all } Z \text {. }\end{array}$ \\
\hline $\begin{array}{l}\text { [Cubit } 13+\text { convention] } \\
\text { double coordx(num_nodes) } \\
\text { double coordy(num_nodes) (2D/3D only) } \\
\text { double coordz(num_nodes) (3D only) }\end{array}$ & $\begin{array}{l}\text { Real arrays holding the } \mathrm{X}, \mathrm{Y} \text {, and } \mathrm{Z} \text { coordinates of the } \\
\text { vertices, if applicable. }\end{array}$ \\
\hline
\end{tabular}

Cubit 13 introduced a new convention to the EXODUS II file which includes different ordering of variables in the files, different variable names. Files written by Cubit 12 lump the vertex coordinates together into one large variable named "coord". Files written by Cubit 13 separate the vertex coordinates into different coordinate variables depending on the number of dimensions present in the mesh: "coordx", "coordy", and "coordz". The converter tool internally checks how many coordinate variables are present in the file so that the coordinates are handled correctly.

\subsubsection{Data Section}

The "data" section contains the values of the arrays declared in the "variables" section of the file. The naming convention is identical to that in Table 3. 


\subsection{Conversion Process}

The following pseudo-code describes the conversion process. The procedure is further described in the following sections.

\section{Check input arguments \\ -Makes sure the file exist and have proper extension (.e, .exo) \\ -Creates the output and report files \\ Open EXODUS II file as a NetCDF data library \\ - 'nc_open' command opens an existing EXODUS II file \\ Retrieve general information about the mesh \\ -'nc_inq' with no data section attached returns the length of each section \\ Retrieve dimensions \\ -'nc_inq_dimid' returns the ID of a dimension listing \\ -'nc_inq_dimname' is used to make sure the proper variable is being queried \\ -'nc_inq_dimlen' returns the value stored at a known ID}

Retrieve Variable IDs

-Based on the dimensional values retrieved, the converter loops through variables

-Finds all needed variable ID's that will be used to retrieve data

-'nc_inq-varid' gives the variable ID

-'nc_inq_varname' is used to check that the proper variable is being queried

\section{Write Coordinates}

-In order to save memory, the coordinate array is written to the output file

-'nc_get_var1_double' is used to pull out one data pt. of type 'double'

-Uses Variable IDs and dimension values to pull out individual data points

-Data is stored in arrays with lengths found in the dimension section

-'nc_get_var1_int' is used to pull out one data pt. of type 'int'

\section{Write Other Info to Output}

-Once all data points have been retrieved and tested for boundary conditions the points are accessed once again in order to write them to the output

\section{Remap Connectivity}

-While only necessary for some element types, a remapping function is called. This second function changes the order that the nodes of each element are written to the output file.

\section{Boundary Information}

-Since the individual data pts. already have been checked for boundary conditions the number of bounds is already known along with all required information.

\section{Figure 7. Description of the conversion process}

\subsubsection{Command Line Input and Output File Creation}

The converter reads the command line argument(s) to check that valid EXODUS II inputs exist and have the correct file extension (.e or .exo). It also creates the file handles for the final ascii mesh and the report summary. The name of both of these documents is based on the EXODUS II input file prefix. Given any input file name, the converter names the output files by reading the character string of the input name, removing the EXODUS II file extension, and replacing it with ".ascii" or "_REPORT.txt". The 
converter can process files outside of the current working directory, but output files are created in the same directory as the input file, not the current working directory.

\subsubsection{Reading the Dimensions}

The converter opens the EXODUS II file as a NetCDF data library using the nc_open command. The converter uses NetCDF commands (see Error! Reference source not found.) to parse through the dimension section and retrieve the information needed to initialize array and set loop variables later on for the variable section. The key information queried here is the number of dimensions, number of element blocks in the mesh, total number of nodes, number of sidesets, number of elements held in each block, and the number of nodes per element in each block. These values are written to the ascii file.

\subsubsection{Reading the Variables}

Using the dimensions retrieved from the dimension section, the converter sets loops to collect the IDs of each block, sideset, and connectivity variable. An ID is essentially an "address" in the file. In order to ensure the correct variable is read, a variable name check is implemented in each major variable retrieval loop.

\subsubsection{Writing Coordinates and Basic Block Information}

At this point, the location and size of important data has been determined. The converter begins process the vertex array point by point rather than as a whole. This point-by-point method takes more time than processing everything at once, but removes any size limitations on the input file. At this stage the vertex coordinates are all written to the output file.

Next, the basic block information is written to the output file: the number of elements in the block, number of boundary elements in the block, number of nodes per element in the block and the equation and interpolation orders of the elements. Each EXODUS II element type is then mapped to a PROTEUS-SN element type, and the PROTEUS-SN element type is written to file along with the block Region ID and the connectivity array.

\subsubsection{Connectivity Remapping}

The connectivity of each element given in the EXODUS II file format is based on EXODUS II element type ordering [1], shown in Figure 8. However, each EXODUS II element type must be mapped via the converter to a PROTEUS-SN element type as shown in Table 4. For element types with different vertex ordering, the connectivity array is changed in order to follow the PROTEUS-SN convention.

Note that CIRCLE, SPHERE and SHELL(3D) EXODUS II element types are not supported as they are not realistic for neutronics simulations. WEDGE may be added in the future although it is not currently supported. Appendix B contains figures that define the element types and node ordering supported by PROTEUS. 


\subsubsection{Boundary Information}

During the data retrieval stage, each element was tested to see if it contained a boundary surface. These elements are then written out to the output file followed by the array of sideset IDs to which each element belongs. Finally, the converter prints a list sideset IDs corresponding to the boundary elements, as well as the element number within the block and the reference surface number containing the boundary surface. 
Node Ordering for Standard Element Types

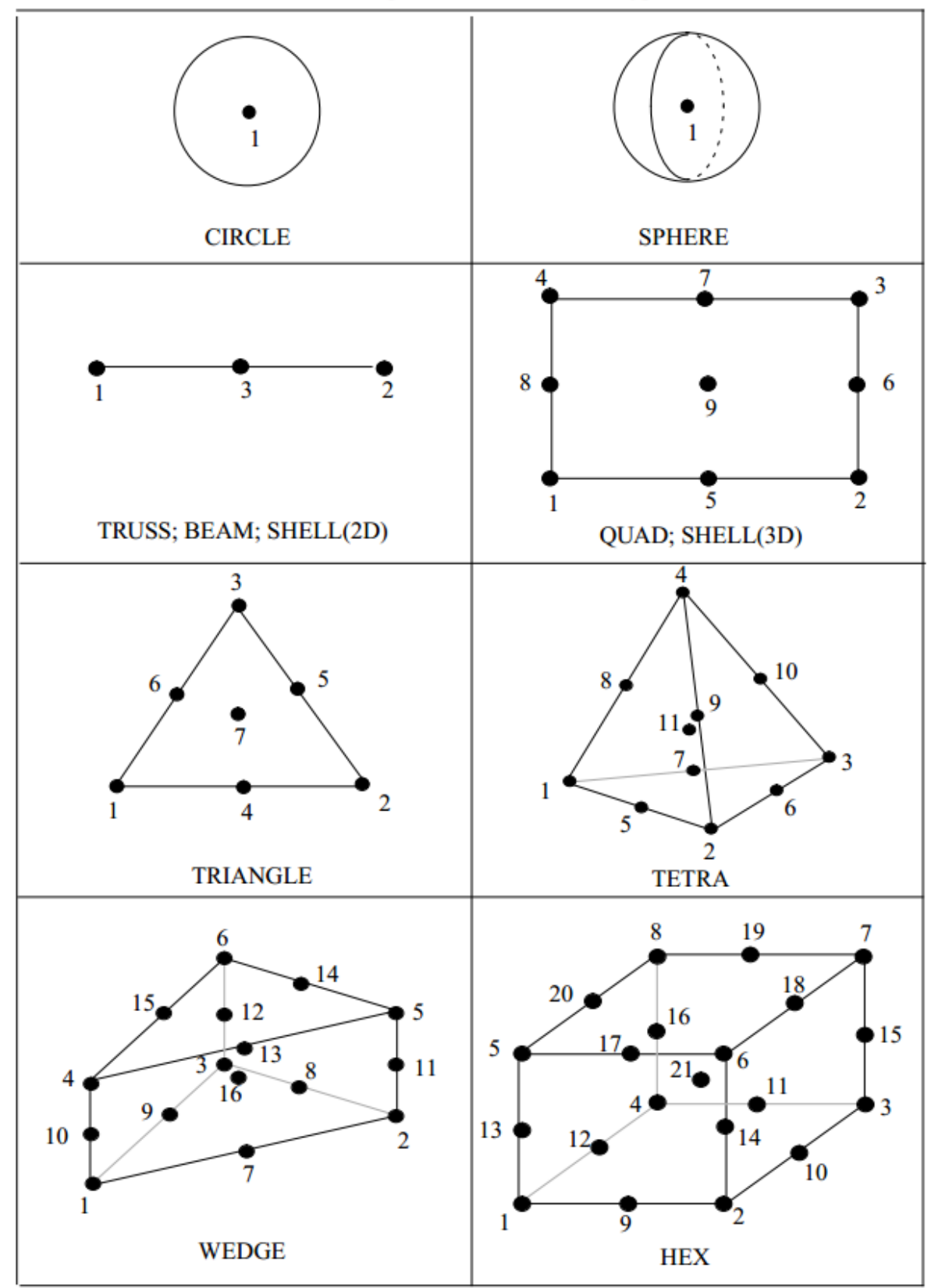

Figure 8. EXODUS II element types and node ordering, courtesy Ref [1].

Table 4. Listing of supported Cubit/EXODUS II element types and node remapping to PROTEUS element type.

\begin{tabular}{|c|c|c|c|c|}
\hline $\begin{array}{c}\text { Cubit } \\
\text { Element } \\
\text { Type }\end{array}$ & $\begin{array}{c}\text { EXODUS II } \\
\text { Element } \\
\text { Type }\end{array}$ & $\begin{array}{c}\text { EXODUS II to Ascii Node } \\
\text { Remapping } \\
\text { (Order of EXODUS II Nodes } \\
\text { listed in Ascii format) }\end{array}$ & $\begin{array}{c}\text { PROTEUS } \\
\text { Element Type }\end{array}$ & $\begin{array}{c}\text { Element } \\
\text { Order }\end{array}$ \\
\hline
\end{tabular}




\begin{tabular}{|c|c|c|c|c|}
\hline $\begin{array}{l}\text { Cubit } \\
\text { Element } \\
\text { Type }\end{array}$ & $\begin{array}{l}\text { EXODUS II } \\
\text { Element } \\
\text { Type }\end{array}$ & $\begin{array}{l}\text { EXODUS II to Ascii Node } \\
\text { Remapping } \\
\text { (Order of EXODUS II Nodes } \\
\text { listed in Ascii format) }\end{array}$ & $\begin{array}{c}\text { PROTEUS } \\
\text { Element Type }\end{array}$ & $\begin{array}{c}\text { Element } \\
\text { Order }\end{array}$ \\
\hline BAR & TRUSS/BEAM & No remapping & BAR & 1 \\
\hline BAR2 & TRUSS/BEAM & No remapping & LAGRANGE BAR & 1 \\
\hline BAR3 & TRUSS/BEAM & {$[1,3,2]$} & LAGRANGE BAR & 2 \\
\hline $\begin{array}{l}\text { TRI / } \\
\text { TRI3 }\end{array}$ & TRIANGLE & No remapping & $\begin{array}{l}\text { LAGRANGE TRI* } \\
\text { (or TRIANGLE) }\end{array}$ & 1 \\
\hline TRI6 & TRIANGLE & {$[1,4,2,6,5,3]$} & LAGRANGE TRI & 2 \\
\hline QUAD & QUAD & {$[1,2,4,3]$} & LAGRANGE QUAD & 1 \\
\hline QUAD4 & QUAD & {$[1,2,4,3]$} & LAGRANGE QUAD & 1 \\
\hline QUAD8 & QUAD & {$[1,5,2,6,3,7,4,8]$} & QUADRILATERAL & 2 \\
\hline QUAD9 & QUAD & {$[1,5,2,8,9,6,4,7,3]$} & LAGRANGE QUAD & 2 \\
\hline $\begin{array}{l}\text { TETRA / } \\
\text { TETRA4 }\end{array}$ & TETRA & No remapping & $\begin{array}{l}\text { TETRAHEDRON* } \\
\text { (or LAGRANGE TET) }\end{array}$ & 1 \\
\hline TETRA10 & TETRA & {$[1,5,2,6,3,7,8,9,10,4]$} & TETRAHEDRON & 2 \\
\hline HEX & HEX & {$[1,2,4,3,5,6,8,7]$} & $\begin{array}{l}\text { LAGRANGE BRICK* } \\
\text { (or BRICK) }\end{array}$ & 1 \\
\hline HEX8 & HEX & No remapping & $\begin{array}{c}\text { BRICK* } \\
\text { (or LAGRANGE BRICK) }\end{array}$ & 1 \\
\hline HEX20 & HEX & $\begin{array}{c}{[1,9,2,10,3,11,4,12,13,14,15} \\
16,5,17,6,18,7,19,8,20]\end{array}$ & BRICK & 2 \\
\hline
\end{tabular}

*An asterisk indicates that the PROTEUS-SN serendipity and Lagrange linear elements are equivalent for this case (identical node ordering).

\subsection{Output File Descriptions}

\subsubsection{ASCII File Format}

The file format for the ascii file is located in Appendix A. The ascii file contains the data found in the EXODUS II file only reordered and reformatted. This new format consists of three sections - 
information regarding the mesh as a whole, the entire coordinate table listing, and the individual block data.

The general mesh information is always located on the first line and states the total number of vertices in the mesh, the number of dimension, the total number of blocks that are present in the mesh, and lastly an unused integer set to zero for later use.

The second section is a list of all coordinates in the mesh ordered by dimension with all $X$ dimension coordinates being listed first, then the $Y$ 's, and lastly the $Z^{\prime}$ 's. Only the coordinates listed in the EXODUS II file are printed to the ascii file, for example, in $2 D, X$ and $Y$ vertices only are printed.

The final section holds information regarding each element block. The first line for each block holds the number of elements in the block, the number of boundary conditions, and how many nodes are attached to each element, an unused integer, the order of interpolation, and the equation order. Following this line is a listing of the element type of the entire block and the region name. The connectivity arrays that hold the node ordering are then listed. The connectivity of a given element type may or may not be reordered from the EXODUS II depending on the element type and interpolation order found during the conversion process. The final pieces of information for this section are the sidesets that correspond to the boundary elements found in the side sets, the boundary elements (numbered by element number within the specific element block ), and lastly the boundary surface associated with each boundary element.

\subsubsection{Report File}

For each conversion performed, a report file is written to show the success or failure of each step in the conversion process. While it is easy to read the error immediately from the command line, the report allows for review later on if needed. 


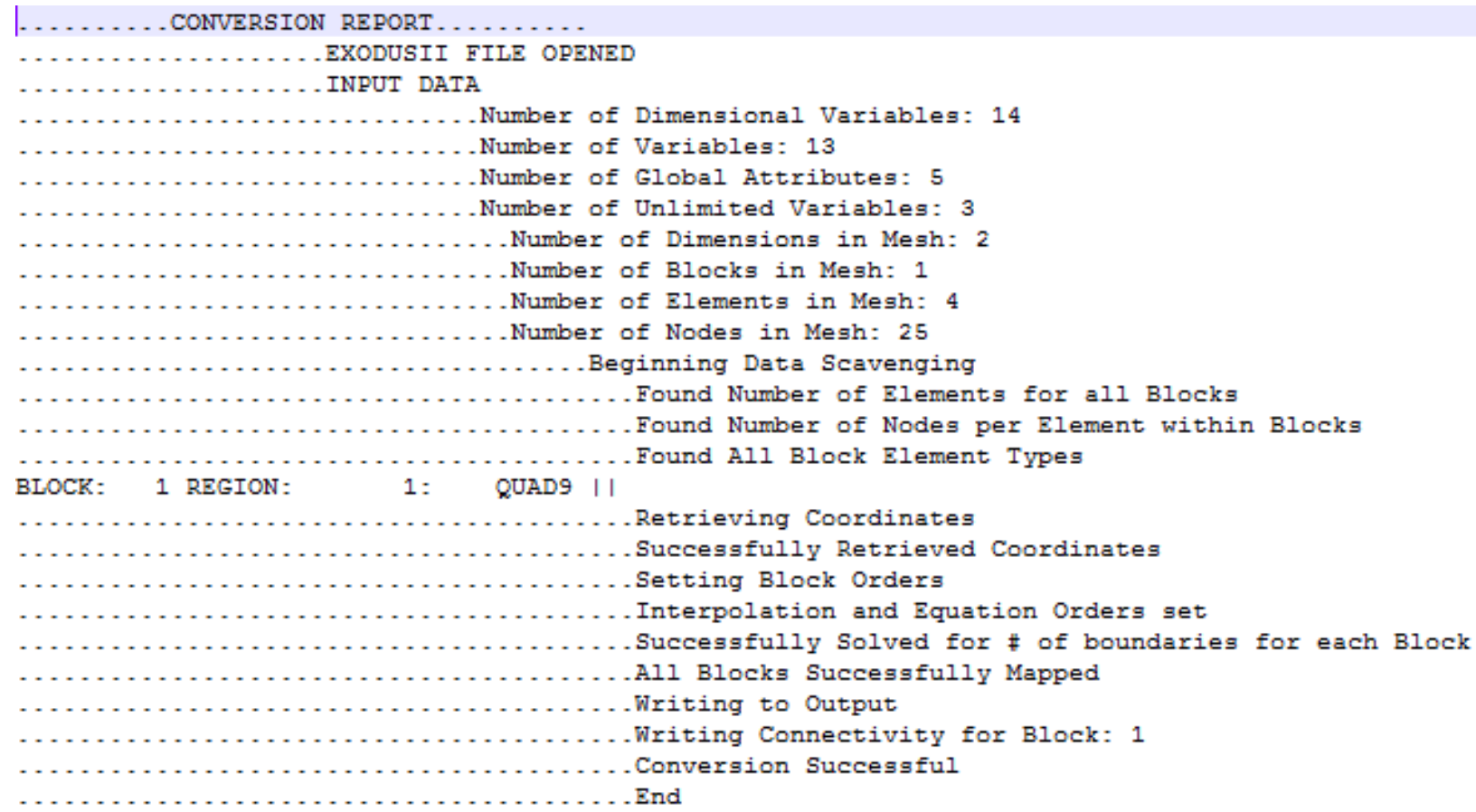

Figure 9. Example of a successful report file.

As shown in Figure 9, the report file echoes the key mesh information as well as each successful step performed in the conversion process. If the conversion process ends early due to an error, the report file can be referenced to see the step where the conversion failed. If an error results from trying to convert an unsupported element type, an incomplete listing of variables in the file, or an error in the node mapping, a clear error message is written in the report file that lists the error encountered and any other information that can help identify the problem. For example, if there is an unknown element type listed in the mesh, then the report announces that there was an unknown element type on a specific block. 


\section{Benchmark Problems}

A series of benchmark tests are provided to make sure that the converter is installed correctly and that the produced ascii meshes are fully compatible with PROTEUS-SN. These tests are separated into two subdirectories under the "verification" directory.

The first directory, "verification/benchmarks" contains several reference EXODUS II and ascii meshes to test whether the source code is working as intended. Invoking the command "make check" runs a script to converts the benchmark meshes and compares the results to reliable reference files. A shell script checks the outputs against the reference files for size and content and writes any differences to the standard output. A description of the benchmark test meshes is given in Table 5 . The tests are intended to cover meshes containing various element types, sizes, and also test different Cubit versions.

Table 5. Benchmark test descriptions "verification/benchmarks" directory.

\begin{tabular}{|c|c|c|c|c|c|}
\hline Bench \# & $\begin{array}{c}\text { File } \\
\text { Extension }\end{array}$ & $\begin{array}{c}\text { Cubit } \\
\text { Version }\end{array}$ & \# of Nodes & $\begin{array}{c}\text { \# of Element } \\
\text { Blocks }\end{array}$ & Element Type(s) \\
\hline 00 &. $\mathrm{e}$ & 12 & 1198 & 4 & QUAD8 \\
\hline 01 &. $\mathrm{e}$ & 12 & 1080 & 3 & TRI3 \& QUAD4 \\
\hline 02 &. $\mathrm{e}$ & 13 & 231600 & 38 & TRI3 \& QUAD4 \\
\hline 03 &. $\mathrm{e}$ & 12 & 535279 & 70 & HEX \\
\hline 04 &. $\mathrm{e}$ & 13 & 13198 & 12 & TRI3 \& QUAD4 \\
\hline 05 &. $\mathrm{e}$ & 13 & 25 & 1 & QUAD9 \\
\hline 06 &. $\mathrm{e}$ & 13 & 81 & 1 & HEX20 \\
\hline 07 &. $\mathrm{e}$ & 13 & 141 & 1 & TRI6 \\
\hline 08 &. $\mathrm{e}$ & 13 & 158 & 1 & TETRA10 \\
\hline 09 &. $\mathrm{exo}$ & 12 & 115494 & 8 & HEX \\
\hline
\end{tabular}

The second test folder, "verification/PROTEUS_Benchmarks", is an optional, extended test-suite to test the compatibility of converter with various versions of Cubit as well as PROTEUS. A series of scripts are invoked that (1) create various EXODUS II meshes with Cubit, (2) convert each mesh to ascii, and (3) test the mesh with PROTEUS-SN. These tests are invoked by the command "make verify". Remember that to be able to run these tests the variables CUBIT_12X, CUBIT_13X and PROTEUS_X must be correctly defined in the Makefile prior to typing the command. 
Table 6. List of Cubit journal files and the elements types to be generated on-the-fly for testing converter compatibility with Cubit and PROTEUS. Each journal file leads to the creation of multiple files used in testing the converter.

\begin{tabular}{|c|c|}
\hline CUBIT Journal File Name & Elements tested \\
\hline \multirow{3}{*}{ generate_TRI.jou } & TRI \\
\cline { 2 - 2 } & TRI3 \\
\hline \multirow{3}{*}{ generate_TETRA.jou } & TRI6 \\
\cline { 2 - 2 } & TETRA \\
\cline { 2 - 2 } & TETRA4 \\
\hline \multirow{5}{*}{ generate_QUAD.jou } & TETRA10 \\
\cline { 2 - 2 } & QUAD \\
\cline { 2 - 2 } & QUAD4 \\
\cline { 2 - 2 } & QUAD8 \\
\hline \multirow{3}{*}{ generate_HEX.jou } & QUAD9 \\
\cline { 2 - 2 } & HEX \\
\cline { 2 - 2 } & HEX8 \\
\hline
\end{tabular}




\section{Summary}

A new EXODUS II finite element mesh converter has been developed to convert EXODUS II meshes (typically created by Cubit) to PROTEUS-SN ascii mesh format. The conversion program replaces an old converter which had limitations on mesh size and compatibility issues with Cubit. The new program works with different versions of Cubit (any version 12+) and has no limitation on EXODUS II file size. Error checking, error reporting and usage syntax have also been greatly improved. The converter is limited to element types supported by PROTEUS-SN (a subset of those produced by Cubit).

For questions, assistance, or to obtain the converter, please contact nera-software@anl.gov. 


\section{References}

1. Schoof, Larry A. and Yarberry, Victor R., "EXODUS II: A Finite Element Data Model" Sandia National Laboratories, Computational Mechanics and Visualization Department, November, 1995.

2. Shemon, E. R., M. A. Smith, C.H. Lee, and A. Marin-Lafleche, "PROTEUS-SN User Manual", ANL/NE14/6, Argonne National Laboratory, June 30, 2014.

3. Russ Rew, Glenn Davis, Steve Emmerson, Harvey Davies, Ed Hartnett, Dennis Heimbigner and Ward Fisher, “NetCDF Documentation” UCAR \& UCP , Unidata, 2008.

4. Ted Blacker, Randy Morris, "Cubit 13.1 User Documentation”, Sandia National Laboratories, Albuquerque, NM, 2012. 


\section{APPENDIX A}

Figure A1. PROTEUS-SN ascii file format.

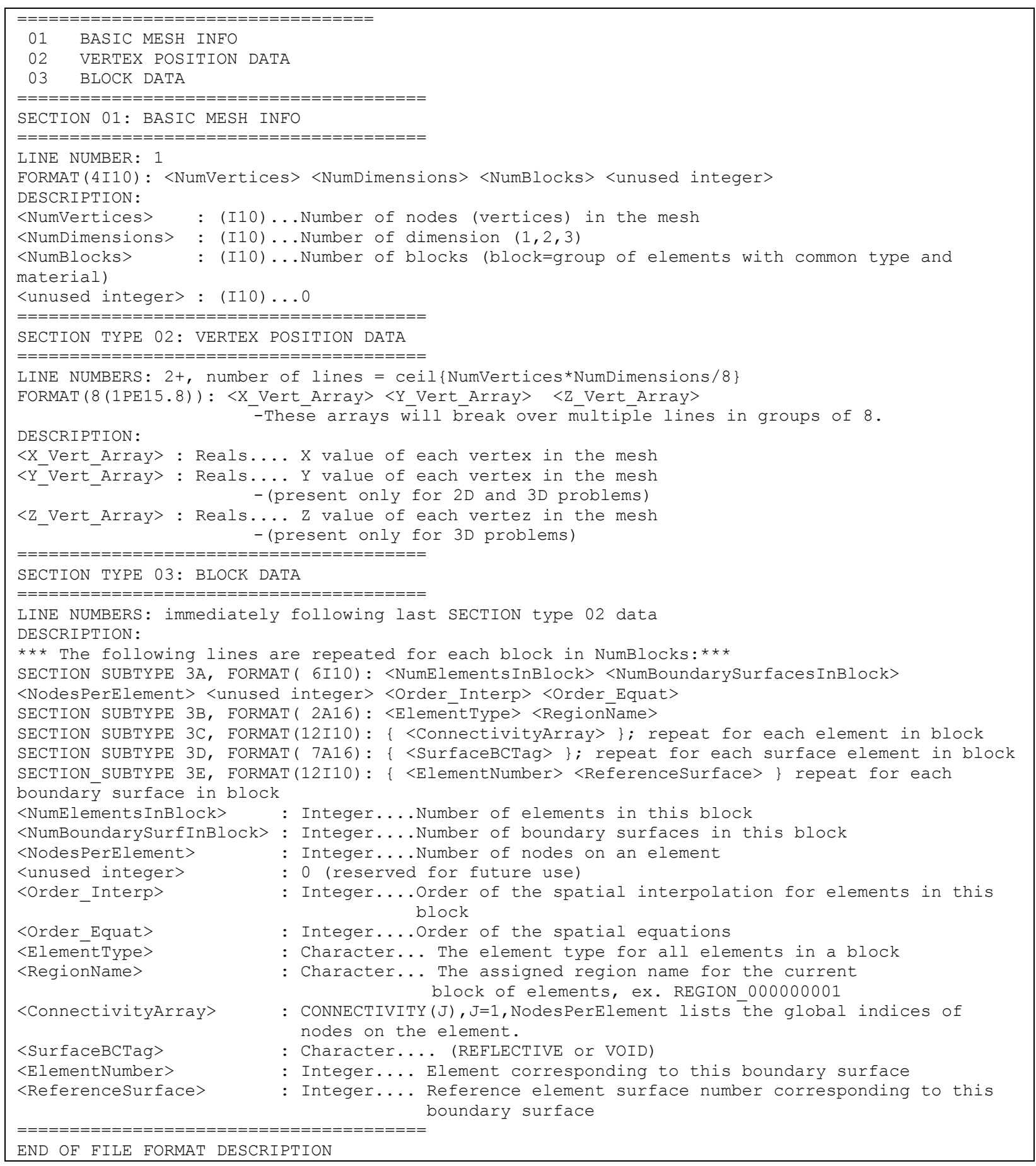




\section{APPENDIX B}

The following figures depict the various element types supported by PROTEUS-SN, including their node ordering.

Cyan color indicates the reference surface numbering for each element.

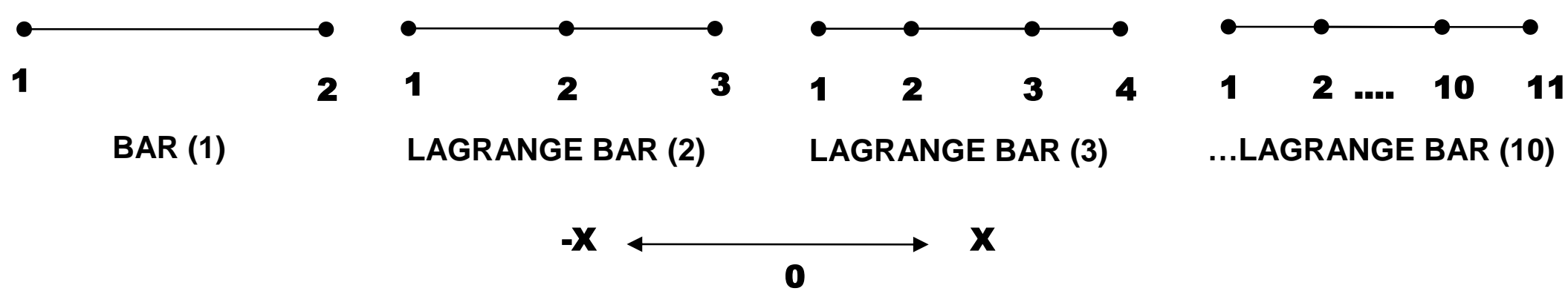

Figure B.1. PROTEUS 1-D Finite Element Vertex Layout. 


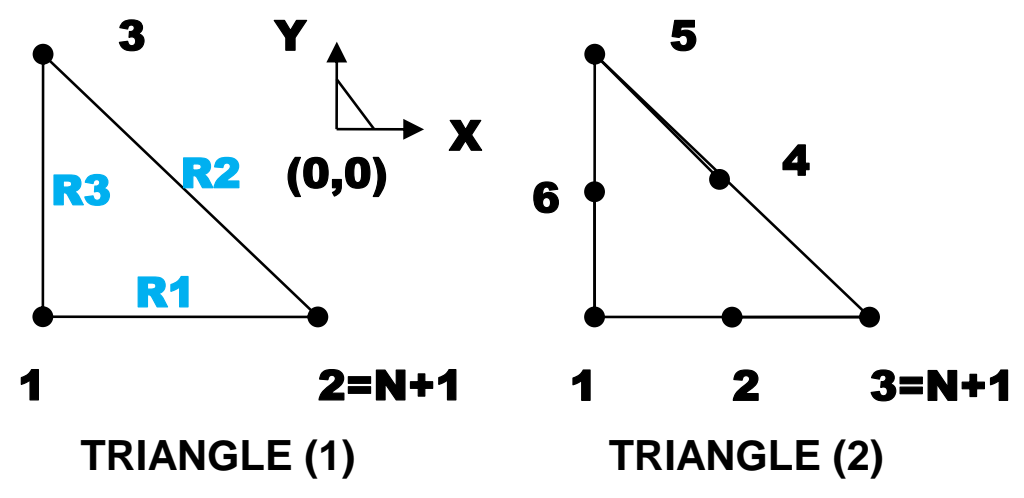

Figure B.2. PROTEUS 2-D Triangular Serendipity Finite Element Vertex Layout.
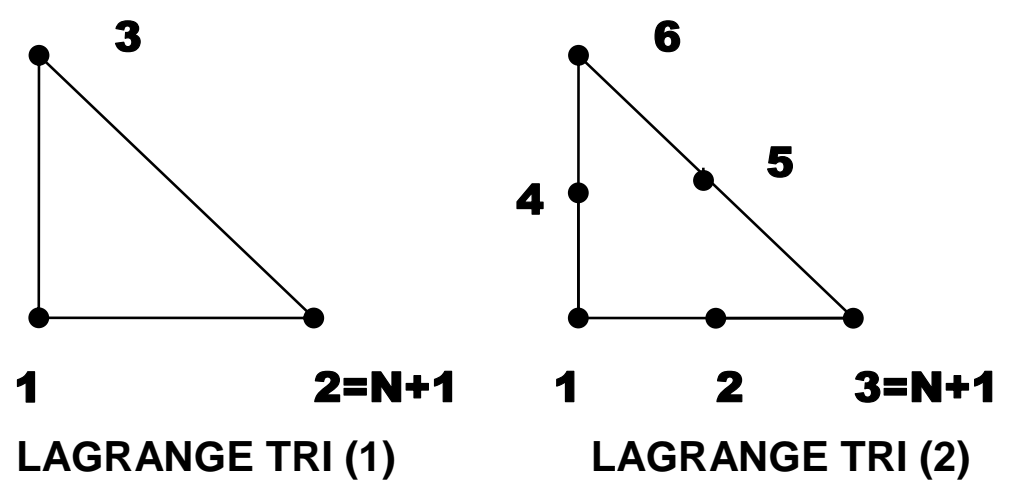
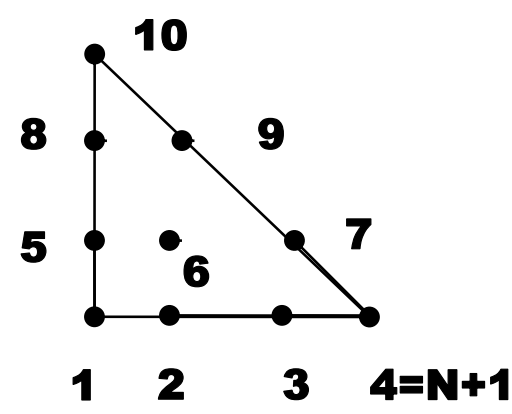

LAGRANGE TRI (3)

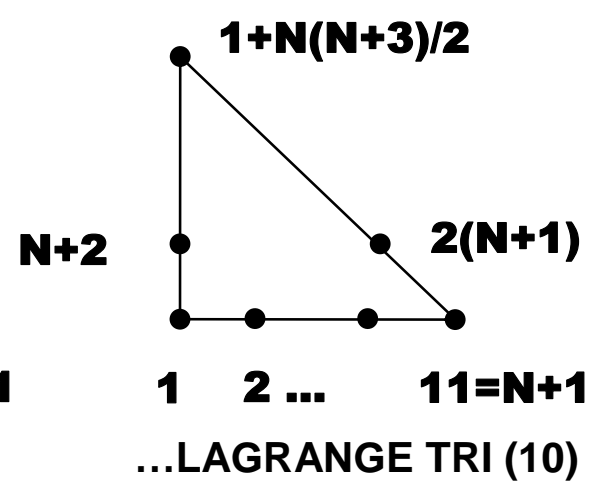

...LAGRANGE TRI (10)

Figure B.3. PROTEUS 2-D Triangular LaGrange Finite Element Vertex Layout. 

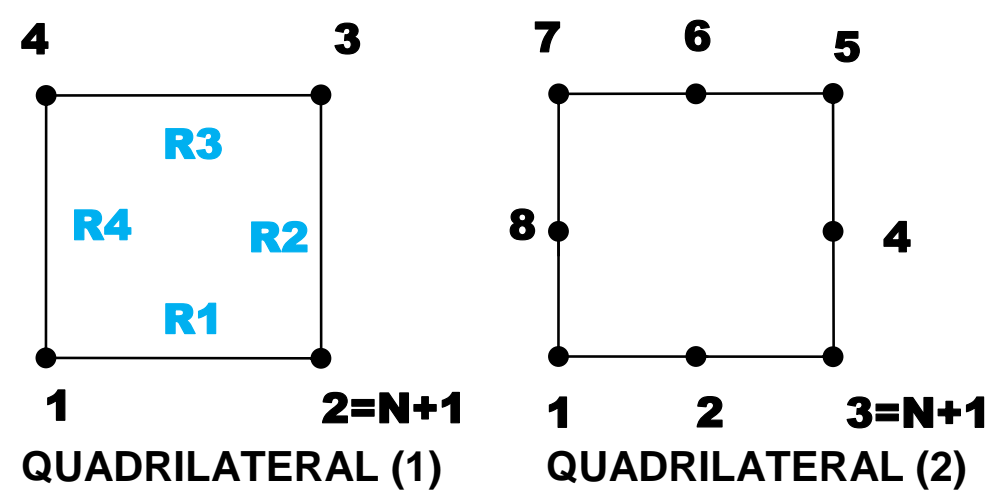

Figure B.4. PROTEUS 2-D Quadrilateral Serendipity Finite Element Vertex Layout.
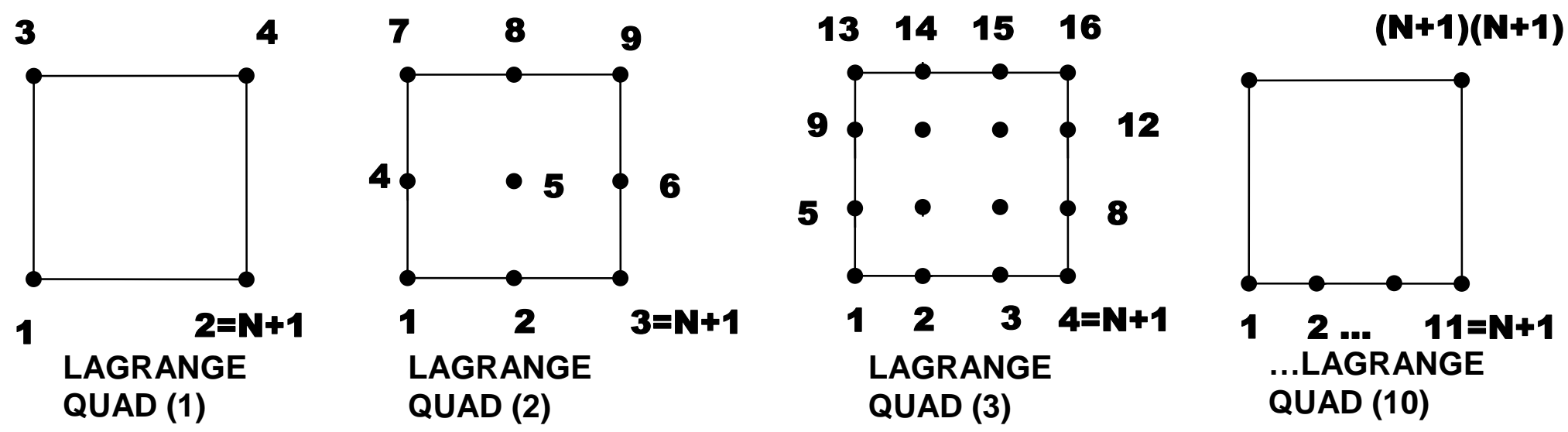

Figure B.5. PROTEUS 2-D Quadrilateral LaGrange Finite Element Vertex Layout. 


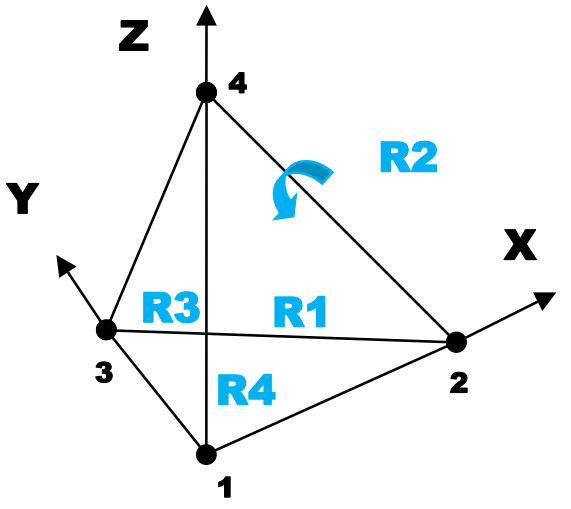

TETRAHEDRON (1)

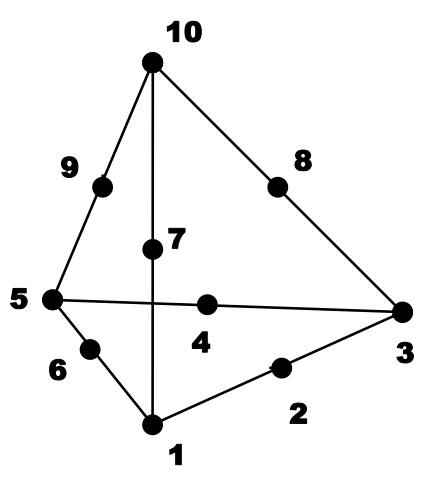

TETRAHEDRON (2)

Figure B.6. PROTEUS 3-D Tetrahedral Serendipity Finite Element Vertex Layout.

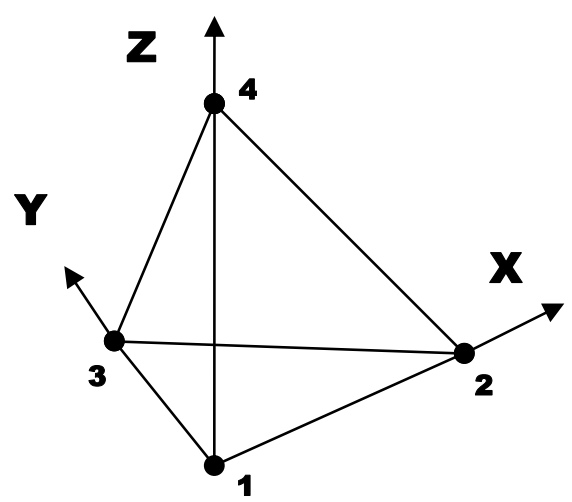

LAGRANGE TET (1)

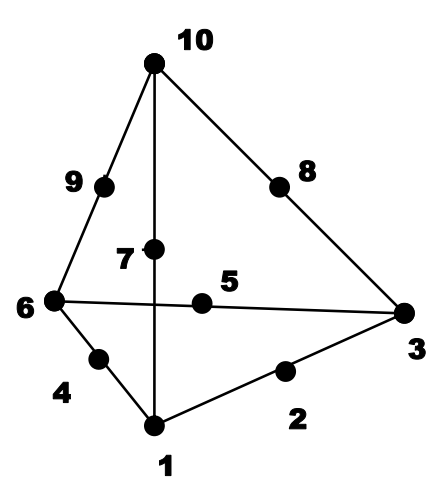

LAGRANGE TET (2)

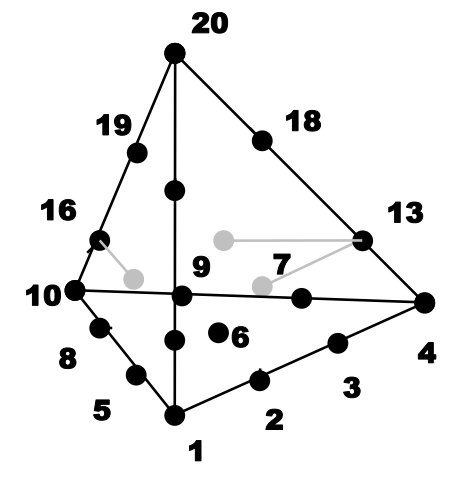

LAGRANGE TET (3)

Figure B.7. PROTEUS 3-D Tetrahedral LaGrange Finite Element Vertex Layout. 


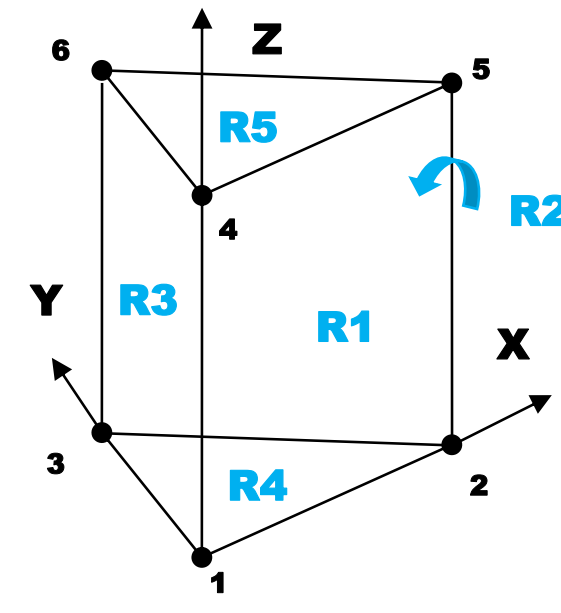

PRISMATIC (1)

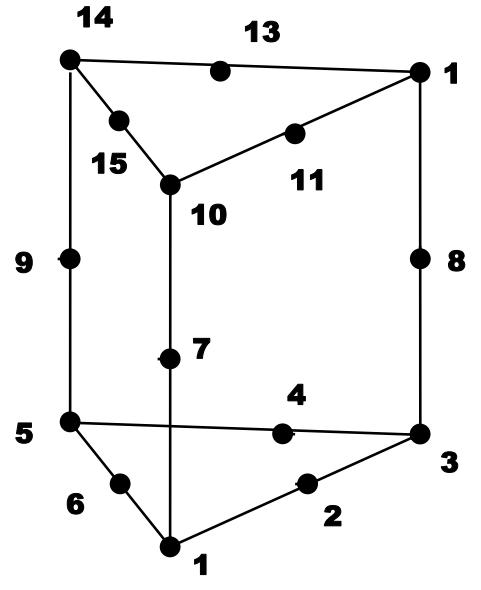

PRISMATIC (2)

Figure B.8. PROTEUS 3-D Wedge (Prism) Serendipity (Natural) Finite Element Vertex Layout.

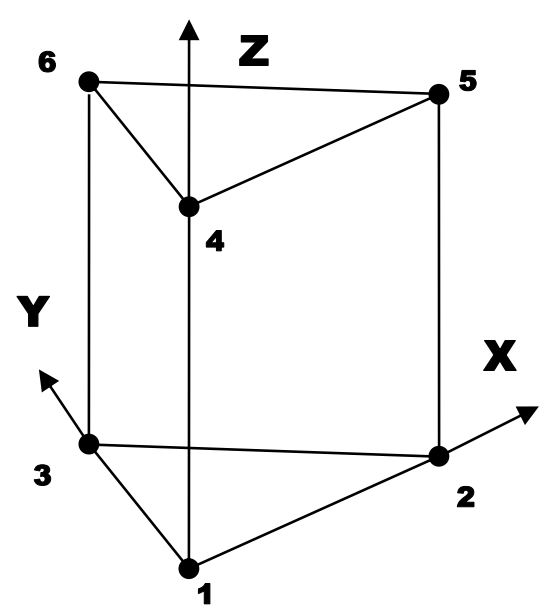

LAGRANGE PRISM (1)

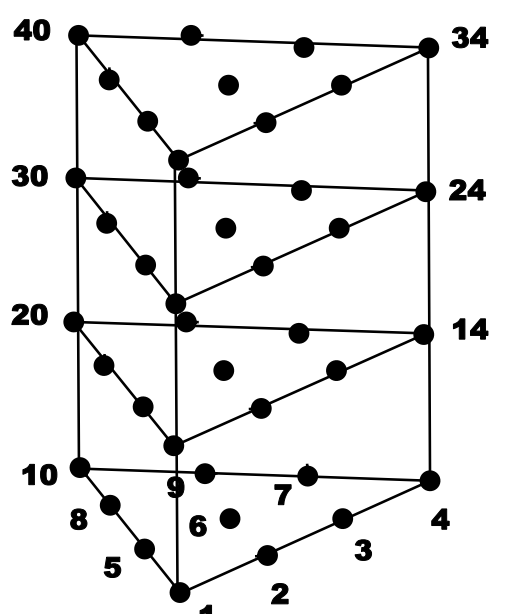

LAGRÁNGE PRISM (3)

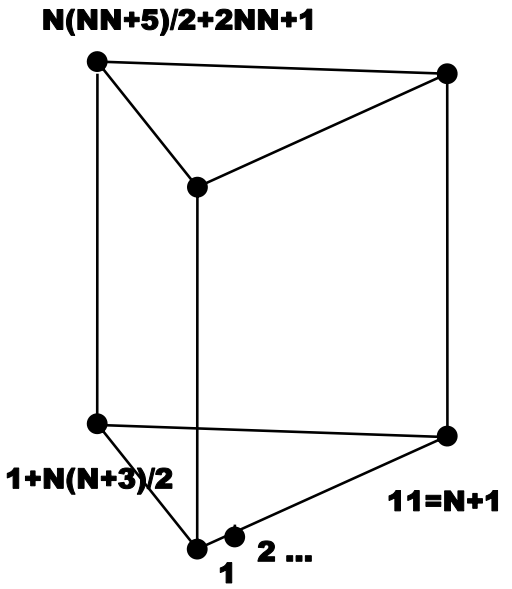

...LAGRANGE

PRISM (10)

Figure B.9. PROTEUS 3-D Wedge (Prism) LaGrange Finite Element Vertex Layout. 

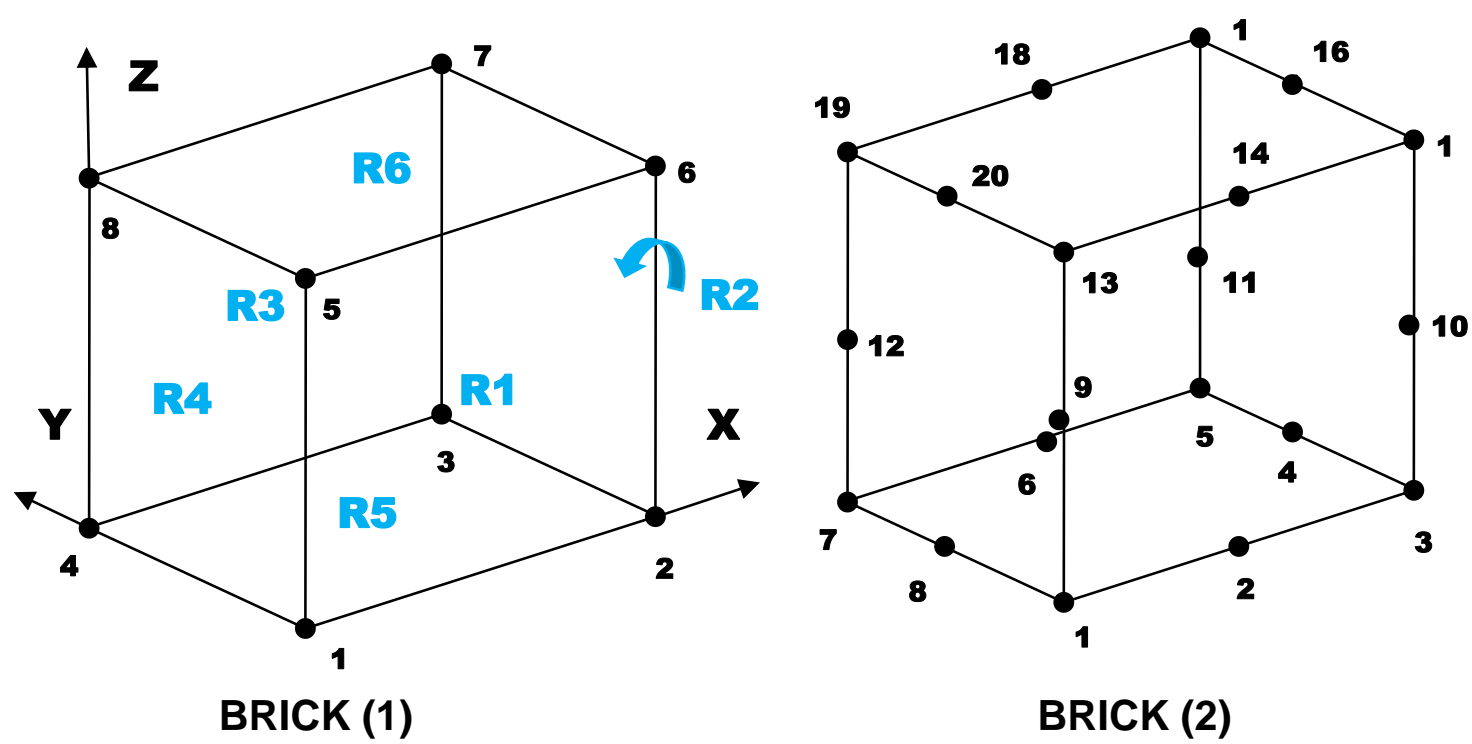

Figure B.10. PROTEUS 3-D Hexahedral Serendipity Finite Element Vertex Layout. 


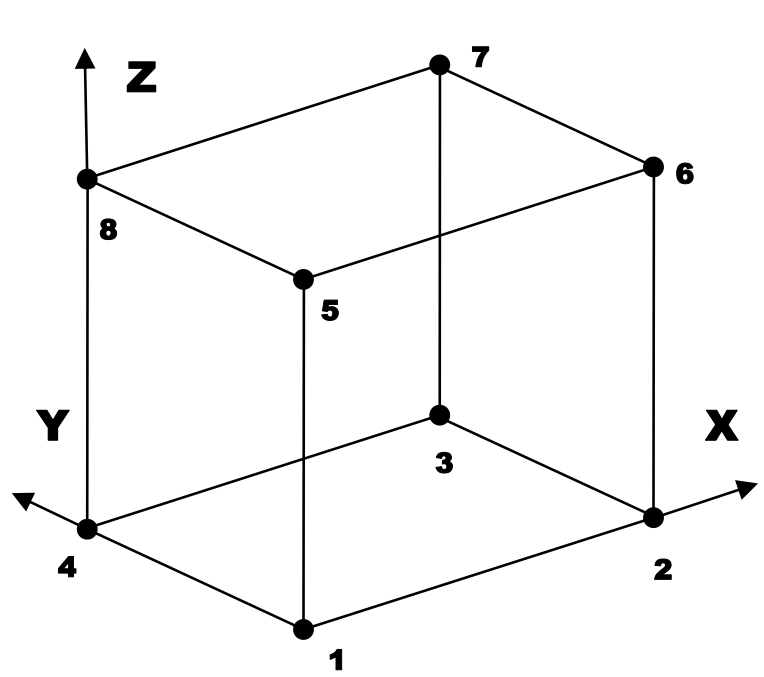

LAGRANGE BRICK (1)

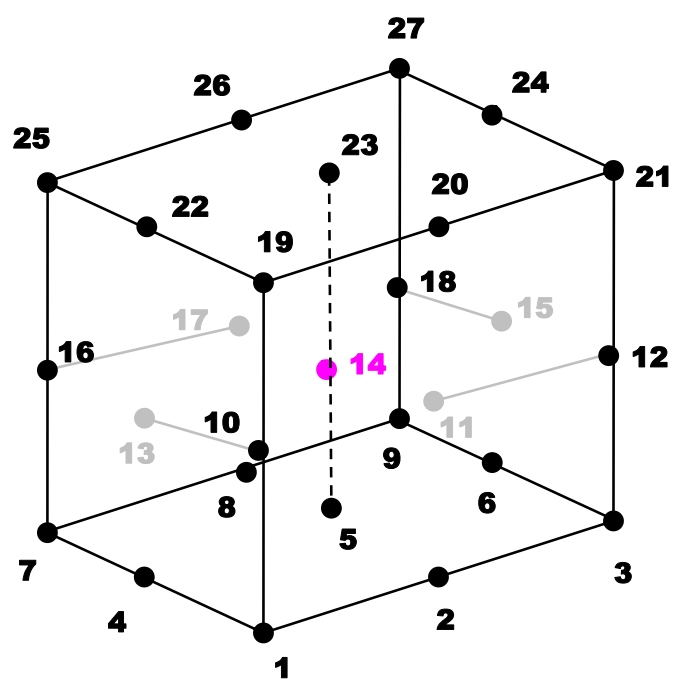

LAGRANGE BRICK (2)

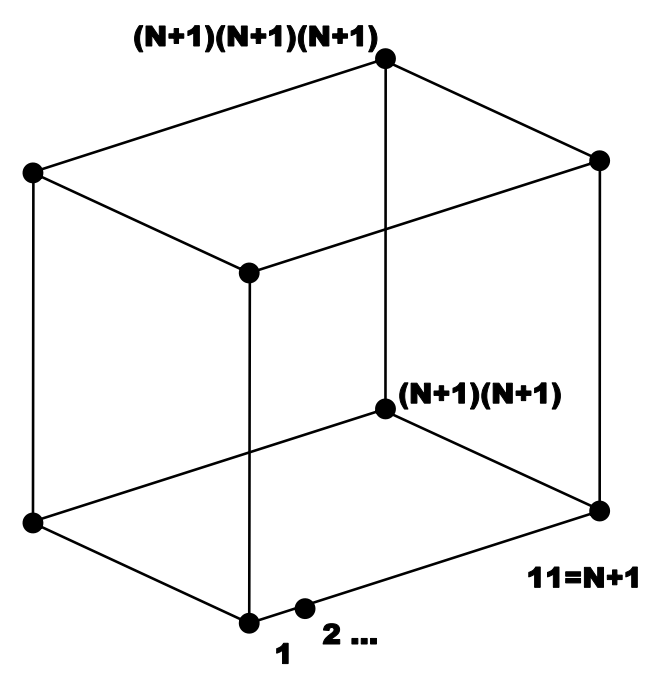

...LAGRANGE BRICK (10)

PROTEUS 3-D Hexahedral LaGrange Finite Element Vertex Layout. 


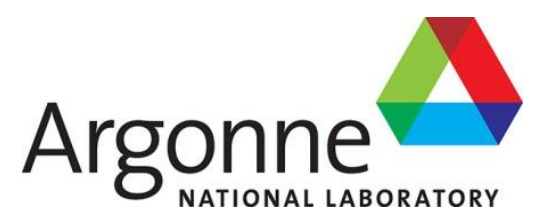

Nuclear Engineering Division

Argonne National Laboratory

9700 South Cass Avenue, Bldg. 208

Argonne, IL 60439-4842

www.anl.gov 